\title{
Suicidal ideations and suicide attempts prior to admission to a psychiatric hospital in the first six months of the COVID-19 pandemic: interrupted time-series analysis to estimate the impact of the lockdown and comparison of 2020 with 2019
}

Christoph Hörmann, Annatina Bandli, Anna Bankwitz, Mateo De Bardeci, Annia Rüesch, Tania Villar De Araujo, Erich Seifritz, Birgit Kleim and Sebastian Olbrich

\section{Background}

There is a substantial burden on global mental health as a result of the Coronavirus disease 2019 (COVID-19) pandemic that has become putting pressure on healthcare systems. There is increasing concern about rising suicidality consequential to the COVID-19 pandemic and the measures taken. Existing research about the impact of earlier epidemics and economic crises as well as current studies about the effects of the pandemic on public mental health and populations at risk indicate rising suicidality, especially in the middle and longer term.

\begin{abstract}
Aims
This study investigated the early impact of the COVID-19 pandemic on suicidality by comparing weekly in-patient admissions for individuals who were suicidal or who attempted suicide just before admission, for the first 6 months after the pandemic's onset in Switzerland with corresponding 2019 control data.
\end{abstract}

\section{Method}

Data was collected at the Psychiatric University Hospital of Zurich. An interrupted time-series design was used to analyse the number of patients who were suicidal.

\section{Results}

Instead of a suggested higher rate of suicidality, fewer admissions of patients with suicidal thoughts were found during the first 6-months after the COVID-19 outbreak. However, the proportion of involuntary admissions was found to be higher and more patients have been admitted after a first suicide attempt than in the corresponding control period from 2019.

\section{Conclusions}

Although admissions relating to suicidality decreased during the pandemic, the rising number of patients admitted with a first suicide attempt may be an early indicator for an upcoming extra burden on public mental health (and care). Being a multifactorial process, suicidality is influenced in several ways; low in-patient admissions of patients who are suicidal could also reflect fear of contagion and related uncertainty about seeking mental healthcare.

\section{Keywords}

COVID-19/SARS-CoV-2 coronavirus pandemic; suicidality; attempted suicide; suicide prevention; psychiatric admission.

\section{Copyright and usage}

(c) The Author(s), 2022. Published by Cambridge University Press on behalf of the Royal College of Psychiatrists. This is an Open Access article, distributed under the terms of the creative Commons Attribution-NonCommercial-ShareAlike licence (https://creativecommons.org/licenses/by-nc-sa/4.0/), which permits non-commercial re-use, distribution, and reproduction in any medium, provided the same Creative Commons licence is included and the original work is properly cited. The written permission of Cambridge University Press must be obtained for commercial re-use.

\section{Mental health and suicidality after virus epidemics}

Since the first infections were reported in China in December of $2019,{ }^{1}$ coronavirus disease 2019 (COVID-19) has spread to almost every country on the planet, infected millions of individuals and taken thousands of lives. Containment measures such as lockdowns, curfews, or restrictions on public movement and gatherings reduced public life to a minimum with many in quarantine or experiencing social isolation. There is a global mental health burden from fear of infection and pandemic control measures. A rising number of studies consistently reported sharp increases in the prevalence of severe mental distress, depression, anxiety, posttraumatic stress and disturbed sleep in the general population ${ }^{2-4}$ with an even higher prevalence in healthcare workers. ${ }^{5}$ Described effects of confinement and social isolation are anxiety, post-traumatic stress disorder, depression, addiction and domestic violence, ${ }^{6}$ which could particularly affect individuals with pre-existing disorders, especially in conjunction with un- or undertreatment, missing resilience or lack of social support. ${ }^{7}$ Shortly following the last pandemic - the Spanish influenza in 1918/1919 - elevated suicide rates were reported, which were related to decreased social integration and pandemic fears. ${ }^{8}$ Furthermore, after the 2003 severe acute respiratory syndrome (SARS) outbreak in Hong Kong, rising suicide deaths as well as higher prevalence of general anxiety, social isolation and psychological distress were found in people aged 65 and over. ${ }^{9}$ Social isolation is a well-known risk factor for suicidality ${ }^{10}$ and may explain to some degree why suicidality rates rise after global virus pandemics.

\section{Economic crisis, unemployment and suicidality}

Unemployment is another important risk factor for suicidality that is associated with a two to three times higher relative risk of dying by suicide. ${ }^{11}$ Relating unemployment rates with suicide rates from 63 countries following the 2008 economic crisis results in an estimated $20-30 \%$ higher relative risk of suicide in people who were unemployed. Higher effects were found in countries with low baseline unemployment. Additionally, a rise in suicide rate preceded a rise in unemployment by 6 months. ${ }^{12}$ Applying this to a scenario, that assumes 24.7 million jobs were lost worldwide as an impact of the COVID-19 pandemic this would mean an increase of about 
9570 suicides. $^{13}$ (According to the International Labour Organization, 33 million people became unemployed in 2020, not including those who shifted into inactivity. ${ }^{14}$ ) In Switzerland, the number of unemployed individuals has increased by almost $52 \%$ since August 2019, along with unemployment rates rising from $2.1 \%$ to $3.3 \%$ up to August $2020 .{ }^{15}$ Transferred to the current situation in 2021 it has to be stated that although millions have lost their jobs or were forced to shut down their own business because of COVID- $19,{ }^{8}$ some negative long-term effects such as growing numbers of unemployed or overindebted households have not yet been fully felt. Accordingly, mental health researchers have expressed concern about rising suicidality rates because of the

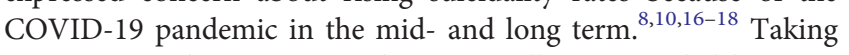
into account that every suicide, statistically, is preceded by more than 20 suicide attempts, ${ }^{19}$ an additional burden for public mental health and suicide prevention is likely to be expected. ${ }^{13}$

\section{Impact of COVID-19 on suicidality}

Although several mental health indicators have been examined for the general public, healthcare workers and individuals with preexisting psychiatric disorders, there is a lack of empirical research on the impact of the COVID-19 pandemic on suicidality. A study from the UK reported a substantial drop in referrals and self-presentations to mental health services after suicide attempts during the first lockdown in April 2020. ${ }^{20}$ A recent study analysing real-time suicide data from 21 (high, upper-middle-income) countries since the beginning of the pandemic found no evidence of an increase in suicide rates in any of them. In 12 areas there was even a significant decrease in suicide rates (i.e. New South Wales, Australia; Alberta and British Columbia, Canada; Chile; Leipzig, Germany; Japan; New Zealand; South Korea; California, Illinois (Cook County), Texas (four counties), USA; Ecuador). ${ }^{21}$

During the initial phase of lockdown, calls to the Swiss helpline 'Die Dargebotene Hand' related to addiction or suicidality increased. This may potentially indicate higher rates of suicidality in Switzerland. ${ }^{22}$ Accordingly, the aim of this study was to investigate the early impact of the COVID-19 pandemic on suicidality and concurrent demands for in-patient mental health services by comparing patient admissions to psychiatric in-patient wards in individuals who are suicidal during the first six months after the COVID19 outbreak with 2019 control data. An interrupted time-series (ITS) design was therefore chosen. ITS is of particular use in evaluating the population-level impact of large-scale healthcare interventions or system-wide shock effects, when randomised data collection is not feasible. ${ }^{23,24}$ The study sample was drawn from a functioning healthcare system with high standards and measures taken for suicide prevention. Nevertheless, as suicide rates have increased after previous pandemics and the measures taken to contain the COVID-19 pandemic have become a public health burden, we hypothesised that the number of admitted suicidal patients would be higher in the first six months after the COVID19 outbreak in Switzerland compared with the corresponding control period from 2019.

\section{Method}

\section{Data collection}

Data was collected at the largest psychiatric university hospital in Switzerland (with a catchment area of approximately 1.2 million). To investigate the impact of the COVID-19 pandemic on weekly admission trends for patients who were suicidal, we collected data for the 6 months starting the day after the first COVID-19 case in Switzerland was confirmed (26 February 2020). Control data was gathered from the corresponding period in 2019. All available entry reports of patients admitted to the in-patient wards of the Clinic for Adult Psychiatry of the University Hospital of Psychiatry Zurich (PUK) were searched for patients with suicidal ideation at admission or those admitted after a suicide attempt. Admissions to youth psychiatry, out-patient treatment programmes or specialised geriatric wards were not included. To align the length of both time series and compensate for an additional leap day, the last day from the 2020 data-set was omitted. After both datasets were truncated after the least full week, 182 days were included in the analyses. To smooth out fluctuations (for example fewer admissions during weekends and holidays) both datasets were comprised on a 7-day basis. The resulting 27 weekly scores for patients who were suicidal at admission and for patients who were admitted after a suicide attempt are indicated by dots in Fig. 1.

De-identified sociodemographic (gender, age, civil status), administrative (number of previous stays, reason for admission, type of referral, involuntary admission) and clinical (ICD-10 diagnoses, suicidality, number of previous suicide attempts) information was extracted for a total of 1591 admissions in 2020 and 1751 admissions in 2019 by the first three authors from the electronic patient filing system. Suicidal tendencies should be addressed at each patients' admission by default as they are a mandatory part of the psychopathological assessment. ${ }^{25}$ Used interchangeably with suicide ideation, suicidality was defined as 'condition that can lead to suicide attempts and suicides. ${ }^{26}$ Suicide attempt was defined as any non-fatal self-injurious act, which is intended to end one's life or for which death is taken into account as a probable outcome. ${ }^{25}$ Involuntary admissions were also coded. According to Article 397a of the Swiss Civil Code, an adult or incapacitated person may be accommodated or detained in a suitable institution for reasons of mental illness, mental weakness, drunkenness, other substance addictions, or severe neglect, unless the necessary personal care can be guaranteed otherwise. ${ }^{27}$ The authors assert that all procedures contributing to this work comply with the ethical standards of the relevant national and institutional committees on human experimentation and with the Helsinki Declaration of 1975, as revised in 2008. All procedures involving human patients were approved by the ethics commission of the canton of Zurich (Req-2019-00056, 05.02.2019). As our study was based on patients' de-identified medical records, the requirement for informed consent was waived.

\section{Statistical analysis}

Our initial hypothesis was that more patients would present with suicide ideation or a recent suicide attempt during the first six months of the COVID-19 pandemic compared with corresponding data from 2019. After noticing that during the early phase of the pandemic fewer patients with suicidal thoughts or recent suicidal attempts were admitted to our clinic, we wanted to examine the significance of this decline and a possible relationship with the uncertainty and measures taken during this (early) phase of the COVID19 pandemic. To estimate the impact of lockdown and other pandemic measures on inpatient admissions of suicidal patients, an ITS design with a segmented regression model was used for admissions during the first 6-months of the COVID-19 pandemic. This was conducted separately, for both patients admitted because of suicidal ideation and those with suicidal behaviour. We defined the event period starting at calendar week 11 and ending at week 19 (13 March and 11 May) and embedded it as a dummy predictor to capture the significance of the first Swiss lockdown. We also added week as a linear predictor to adjust for seasonality and time-varying confounders. This model was also used for a counter check on the 2019 control data and to check for a common trend 
(a)

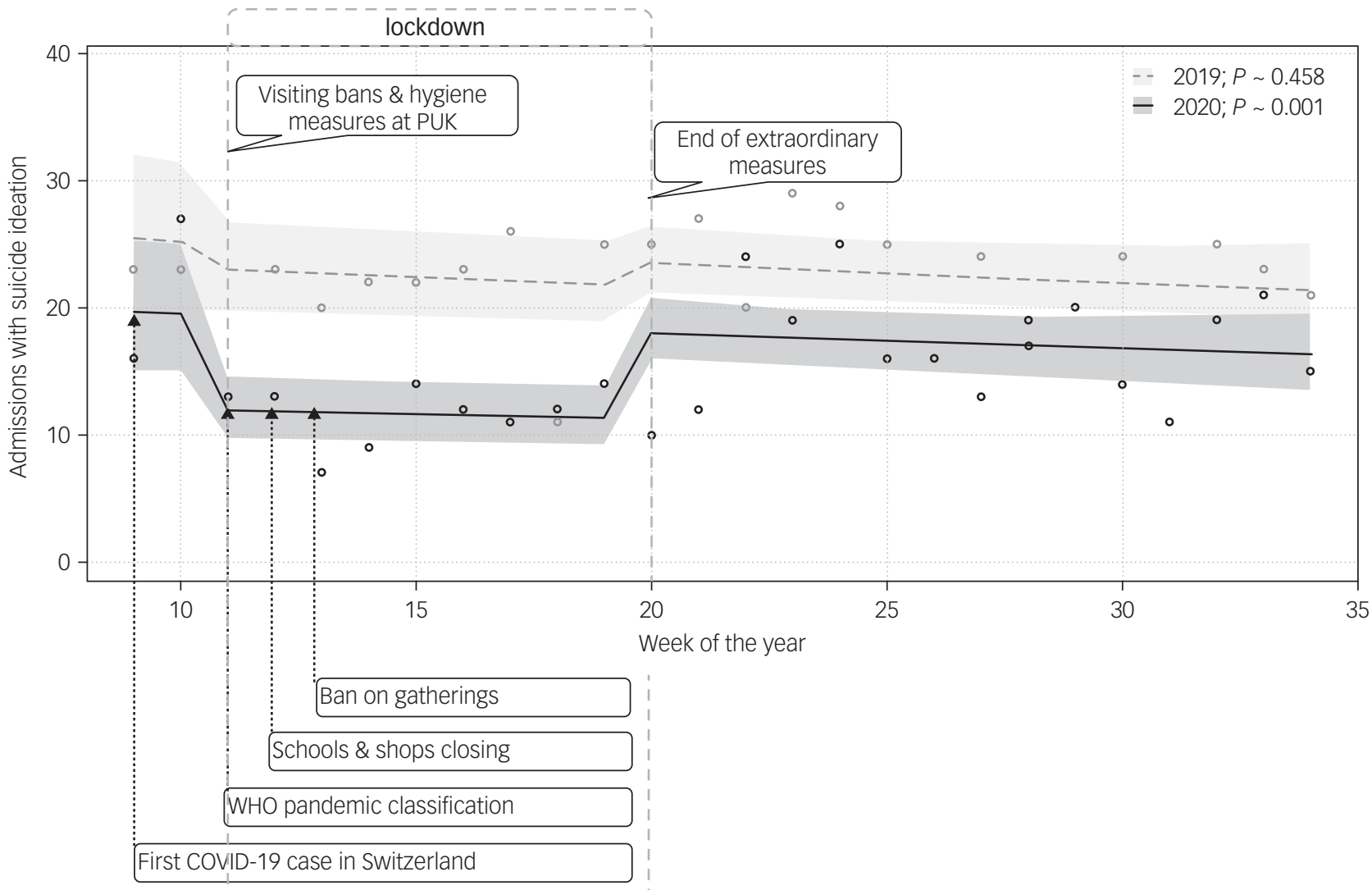

(b)

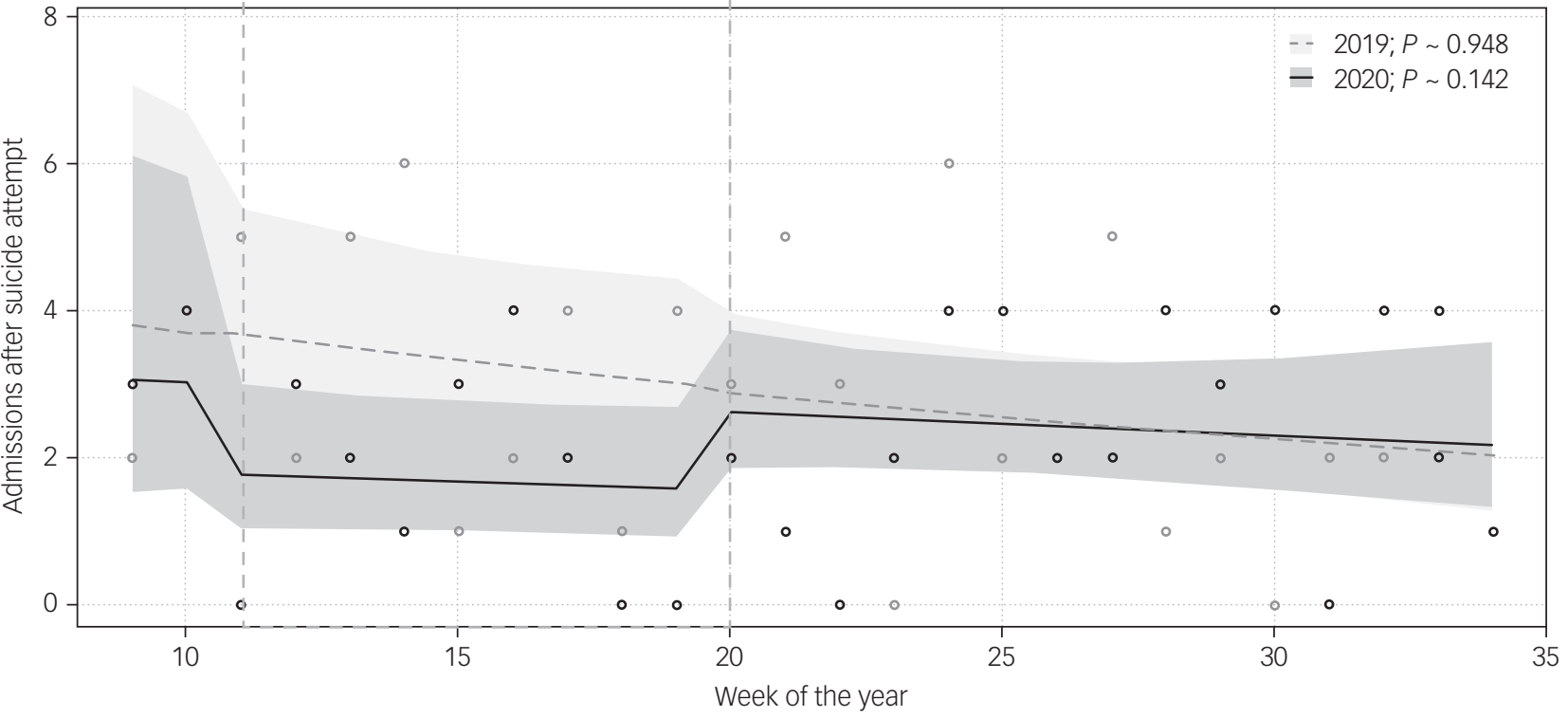

Fig. 1 Admissions of patients who were suicidal at admission (a) or admitted after suicide attempt (b) and the chronology of lockdown-related events and restrictions. Dots represent weekly admission counts for the first 6-months of the COVID-19 pandemic and the corresponding control period from 2019 as indicated.

Horizontal lines depict trends of modeled regressions on admission counts. Grey-shaded areas indicate the corresponding $95 \%$ confidence intervals. Only for the 2020 data, an event-predictor dummy variable representing the lockdown period was used. Presented $P$-values indicate the significance of the event-predictor in the regression model (for interrupted time-series model statistics, see the supplementary Tables). COVID-19, coronavirus disease 2019; PUK, University Hospital of Psychiatry Zurich; WHO, World Health Organization.

by using subtracted counts of 2019 from 2020 as response variable (model statistics for all conducted regression analyses are in the Supplementary Tables; available at http://dx.doi.org/10.1192/bjo. 2021.1072). For the weekly counts of admissions of patients who were suicidal and patients admitted after a suicide attempt a Poisson distribution was assumed. However, the common trend analysis with the differenced counts is based on a quasi-Poisson distribution to adjust for overdispersion. For all time series we 
Table 1 Admissions and suicidal subgroups (26 February to 25/26 August) 2020 versus 2019

\begin{tabular}{|c|c|c|c|c|}
\hline & 2020 & 2019 & Relative change in 2020 compared to 2019 & $\chi^{2}$ (d.f.) sig \\
\hline Total & 1591 & 1751 & & \\
\hline Female patients & 735 & 817 & -0.46 & 0.07 (1) 0.79 \\
\hline Age & & & & $1.33(2) 0.51$ \\
\hline Under 30 years & 388 & 399 & +1.60 & \\
\hline $30-59$ years & 951 & 1077 & -1.73 & \\
\hline 60 years and over & 252 & 275 & +0.13 & \\
\hline Civil status & & & & $3.09(2) 0.21$ \\
\hline Married or in civil union & 186 & 246 & -2.36 & \\
\hline Separated, divorced, widowed & 251 & 269 & +0.41 & \\
\hline Single or unmarried ${ }^{a}$ & 790 & 878 & -0.49 & \\
\hline Involuntary admissions & 634 & 614 & +4.78 & $8.15(1)^{\star \star}$ \\
\hline suicide ideatation subgroup & 402 & 589 & -8.37 & $28.00(1)^{\star * * *}$ \\
\hline Female patient & 211 & 303 & +1.04 & 0.10 (1) 0.75 \\
\hline First psychiatric in-patient admission & 181 & 277 & -2.00 & 0.38 (1) 0.39 \\
\hline Age & & & & 1.50 (2) 0.47 \\
\hline Under 30 years & 136 & 180 & +3.27 & \\
\hline 30-59 years & 228 & 344 & -1.69 & \\
\hline 60 years and over & 38 & 65 & -1.58 & \\
\hline suicide attempt subgroup & 57 & 74 & +0.64 & $0.92(1) 0.34$ \\
\hline Female patients & 29 & 41 & -4.53 & 0.26 (1) 0.61 \\
\hline First-time attempt & 20 & 8 & +24.28 & $11.29(1)^{\star \star \star \star \star}$ \\
\hline
\end{tabular}

conducted Durbin-Watson tests, which showed no evidence for autocorrelation. Statistical analysis and plots were produced with $\mathrm{R}$ v4.1.1. ${ }^{28}$

\section{Results}

Compared with the 2019 data, a non-significant decrease of 9.14\% was observed for the total number of admissions. Table 1 shows sociodemographic and clinical statistics for total in-patient admissions and suicidal subgroups for the 6 months following the COVID-19 pandemic outbreak and the corresponding period in 2019. Patients admitted to PUK during the 6 months after COVID-19 outbreak were more often admitted involuntarily and had substantially fewer $(T(3312)=3.78, r=0.07, P<0.001)$ previous hospital admissions (mean 4.22 , s.d. $=8.36$ ) compared with the 2019 control period (mean 5.53, s.d. $=11.28$ ).

Contrasting with our a priori expectations, which were that more patients who were suicidal would be admitted during the period in 2020 with the COVID-19 pandemic, a significant relative reduction of $8.37 \%$ of patients admitted with suicidal ideation was found for the 6-month period in 2020 compared with the 2019 data. Although there seemed to be a reduction in hospital admissions after suicide attempts in absolute numbers, the relative share of total admissions was similar in both periods.

In the 2019 control period, 74 patients were admitted after a recent suicide attempt, whereas 589 patients were identified as suicidal. In the 6 months following the COVID-19 pandemic in 2020 , almost $32 \%$ fewer $(n=57)$ patients were admitted after a suicide attempt and around $23 \%$ less $(n=402)$ with suicidal ideation.

Furthermore, we found that in the 6 months after the COVID19 outbreak, the proportion of patients admitted after a first suicide attempt had more than tripled compared with 2019 (from 10.81\% $(8 / 74)$ to $35.09 \%(20 / 57)$ of admissions after a suicide attempt; $\chi^{2}=11.29$, d.f. $\left.=1 ; P<0.0001\right)$. During the 9 weeks in which lockdown restrictions were in effect in Switzerland (11 Mar to 12 May 2020), only half as many patients were admitted after a suicide attempt compared with the same period the year before $\left(\chi^{2}=3.79\right.$, d.f. $\left.=1 ; P=0.05\right)$.
To analyse the early impact of the COVID-19 pandemic on suicidality-related admissions, three regression analyses were conducted with the same model for both patients with suicidal ideation at admission and patients admitted to hospital because of suicide attempts. Fig. 1 shows the 7-day admission counts of patients who were suicidal between calendar weeks 9 and 34 from 2020, and the 2019 control data. As indicated by the $P$-value in Fig. 1, we could reject the null hypothesis for our model on 2020 suicidal admission counts, which explained $36 \%$ of deviance and indicated a significant $(P<0.001)$ step change of $-37.9 \%$ for admissions with suicidal ideation between week 11 and 19 (rate ratio $(\mathrm{RR})=$ $0.621,95 \%$ CI $0.539-0.716$ ). That suicidal admissions were significantly lower during the lockdown period is also graphically indicated by the white space left in between both trend lines. Moreover, the mean with $95 \%$ confidence interval of suicidal ideation counts in Fig. 1 only overlap before and after the event period. As can be seen in Fig. 1, there was no sudden rise of suicidal admissions associated with loosening of measures after week 20 in 2020.

To remove confounding variables, we used data from 2019 as a control by calculating a new time series by subtracting the values for 2019 from 2020. We did not find evidence against the common trend assumption for suicide ideation. Accordingly, we found the trend for 2019 to be parallel to the trend for 2020 before and after lockdown, supporting thereby the common trend assumption. In this case, we found a step change of $-33 \%(R R=0.670,95 \%$ CI $0.477-0.942$ ) during lockdown, with a $P$-value of 0.04 . Thus, when using 2019 as a control, we still found a significant reduction of admissions for suicide ideation. Corresponding ITS model statistics are available in Supplementary Table 1. Nevertheless, we did not find any evidence of an increase of admissions with suicide ideation.

\section{Discussion}

\section{Principal findings}

In accordance with several authors ${ }^{8,10,16-18}$, who raised concerns about rising suicidality following the outbreak of COVID-19, higher admission rates for patients admitted with suicide ideation or after a suicide attempt were hypothesized for the first 6 months 
in 2020 than in the 2019 control period. For the ITS analyses, we initially assumed an association of such an effect with the implementation of lockdown and restrictions in the canton of Zurich (Switzerland). No such effects could be observed. Contrasting $a$ priori expectations, it was found that compared with 2019, significantly fewer patients had been admitted with suicidal ideation during the 6 months following the COVID-19 outbreak. The finding that compared with 2019 only half as many patients with a recent suicide attempt were admitted in the period between week 11 and 19 of 2020, gives reason to speculate: Is it possible that the lockdown restrictions have (temporarily) led to even lower rates of suicidality? Or is it more likely that fears of contagion might have outweighed some individuals' willingness to seek help for coping with suicidality and mental distress.

The fact that the total number of admissions to PUK decreased during the first six months of the COVID-19 pandemic, while a higher proportion of patients had to be admitted involuntary, may be an indication of a lower willingness to treat. A higher proportion of compulsory admissions as well as an increase in patients with first-time suicidal behaviour during the immediate aftermath of COVID-19 could also suggest that rates of suicidal ideation or attempted suicide have remained unchanged or may be even increased, but that patients who were suicidal were not presenting for mental healthcare. Interestingly a large-scale UK study found that primary care contacts for major physical and mental health conditions had considerably dropped after the introduction of restriction measures and remained low until July 2020. ${ }^{29}$ Another conceivable explanation for the increase in first-time suicide attempts may be that the pandemic and related measures could have altered the mechanisms of certain suicide risk factors. This could have aggravated suicide risk in individuals who were at a much lower risk prior to the pandemic. Pandemic measures such as distance regulations and contact bans may have specifically increased social isolation for individuals with few social contacts in private life but a functional social network at their workplace.

Although helpline calls in Switzerland related to addiction and suicidality increased, ${ }^{22}$ we observed declining suicidality-related admissions, which could mean that some individuals might have tried to cope with suicidality on their own. This could be reflected in a possible increase in actual suicides during the study period. However, the 2020 report of the Zurich cantonal police force revealed a comparable decline in overall suicide attempts and suicides in the first 9 months after the outbreak of the COVID-19 pandemic in Switzerland. ${ }^{30}$ After the suicide rate in Switzerland had been steadily falling for decades, no further decline has been recorded in recent years (the last available official cause of death statistics includes suicides until 2018). ${ }^{31}$ Last but not least, the cantonal priority programme on suicide prevention, which has launched a number of relevant campaigns, actions and measures since 2015, raised public awareness about suicidality and might have helped to prevent suicides. ${ }^{32}$

\section{Comparison with previous studies}

Our observations seem to be consistent with a previous study that reported a general drop in referrals and presentations to primary mental healthcare providers and community health services as well as lower referral rates related to suicidality during lockdown and the early phase of the COVID-19 pandemic in Cambridgeshire and Peterborough, UK. ${ }^{20}$ Decreased suicide numbers during the first 6 months of the COVID-19 pandemic were also found in Tyrol, Austria. ${ }^{33}$ In contrast to a Spanish study, which reported a continuous decline of suicide-related emergency department visits during a very early phase of the COVID-19 pandemic in Madrid, ${ }^{34}$ we neither found a permanent decline nor a sudden rise in patients who were suicidal after stepwise loosening of containment measures in Switzerland. Although there is some evidence that suicide rates repeatedly fall during wars, which is explained by higher social cohesion uniting against a common foe, ${ }^{35}$ we may be better off expecting 'a hidden burden of mental illness, which will become apparent in the months to come ${ }^{20}$ - possibly not only from patients. A recent study of suicide rates in Japan found that monthly suicide rates had increased during July to October 2020, after an initial decline during the first COVID-19 wave. ${ }^{36}$ Whereas the large-scale Swiss corona stress study ${ }^{37}$ found indicators for psychological distress and depression to be moderately increased during the first lockdown in April, their recent follow-up revealed that the proportions of participants reporting maximum stress or experiencing depressive symptoms had increased to $20 \%$ during the second pandemic wave in November $2020 .{ }^{37}$ As main drivers for rising psychological distress, the authors name changes in work, school or education as well as financial losses or fears about the future related to COVID-19. The authors further report a considerable increase in emergency consultations in youth psychiatry, which was estimated to be around $40 \%$ higher than in 2019. ${ }^{37}$ Younger individuals may carry a high burden with current restrictions and social distancing measures; surveying more than 1500 German-speaking individuals, elevated acute stress owing to COVID-19 among younger people was observed. Conversely, the degree of possible self-control and experience of meaning in life, which can both buffer COVID-19 related stress and fears and relieve stress and anxiety associated with COVID19 , only increases with age. ${ }^{38}$

\section{Strengths and limitations}

This paper provides important observations and insights about the impact of the COVID-19 pandemic on population-level suicidality and the associated need for inpatient mental care. The ITS approach used is a robust and powerful tool to evaluate the impact of largescale interventions and is appropriate for assessing changes in routine data after well-defined events. ${ }^{24}$ Our data appears to be robust against common biases such as seasonality and autocorrelation. In addition, we were able to demonstrate a significant effect of the pandemic measures with the common trend model for suicide ideation. One limitation of our study is that only data from one, although large, psychiatric hospital was evaluated. Furthermore, data was only collected at an early phase of the COVID-19 pandemic. The relatively low number of patients reduces the generalisability and statistical significance for admissions after suicide attempts. Further investigation is needed to analyse suicidality and the consequent demand for mental health services during the second wave and subsequent phases of the pandemic as a reintroduction of restrictive measures was necessary because of mutations and high incidences of COVID-19. As people under the age of 18 are underrepresented in our sample, findings are not generalisable to individuals below this age. Older adults might also be underrepresented as admissions to specialised geriatric wards were not included.

\section{Interpretation and implications}

The finding that admissions of patients with suicidal ideations or after a suicide attempt did not increase following the first lockdown in 2020 compared with the control period in 2019 may be counterintuitive. However, this study only sheds light on the very first months following the outbreak of COVID-19. Those observations should thus be interpreted with caution, as the assumption that there was no rise or even a reduction in suicidality is just one of several explanations, as discussed above. Higher rates of compulsory admissions and first-time suicide attempts during the first 6 months 
of the COVID-19 outbreak should be taken as an alarming signal. It has to be hypothesised that in the upcoming year, the numbers will increase and more suicides will occur as a long-term consequence of pandemic measures. To further establish the impact of the COVID19 pandemic and associated measures on suicidality and concurrent demand for mental healthcare more studies are needed. Psychiatric conditions are the result of complex interactions and need time to manifest. Whereas somatic effects of COVID-19 infection are immediately apparent, suicidality and suicide attempts are the result of various biological, social and economic stresses that may occur much later. Thus, the presented results must therefore not be interpreted as an all-clear against a (possible) increase in suicidality in the upcoming months.

Christoph Hörmann (D), Department of Psychiatry, Psychotherapy and Psychosomatics, Psychiatric University Hospital, University of Zurich, Switzerland; Annatina Bandli, Department of Psychiatry, Psychotherapy and Psychosomatics, Psychiatric University Hospital, University of Zurich, Switzerland; Anna Bankwitz, Department of Psychiatry, Psychotherapy and Psychosomatics, Psychiatric University Hospital, University of Zurich, Switzerland; Mateo De Bardeci, Department of Hospital, University of Zurich, Switzerland; Mateo De Bardeci, Department of
Psychiatry, Psychotherapy and Psychosomatics, Psychiatric University Hospital, University of Zurich, Switzerland; Annia Rüesch, Department of Psychiatry, Psychotherapy and Psychosomatics, Psychiatric University Hospital, University of Zurich, Switzerland; Tania Villar De Araujo, Department of Psychiatry, Psychotherapy and Psychosomatics, Psychiatric University Hospital, University of Zurich, Switzerland; Erich Seifritz, Department of Psychitry, PSychotherapy and Psychosomatics, Erich Seifritz, Departmentic Psychiatric University Hospital, University of Zurich, Switzerland; Birgit Kleim Department of Psychiatry, Psychotherapy and Psychosomatics, Psychiatric University Hospital, University of Zurich, Switzerland; and Department of Experimental Psychopathology and Psychotherapy, University of Zurich, Switzerland; Sebastian Olbrich, Department of Psychiatry, Psychotherapy and Psychosomatics, Psychiatric University Hospital, University of Zurich, Switzerland

Correspondence: Christoph Hörmann. Email: christoph.hoermann@uzh.ch

First received 9 Jun 2021, final revision 23 Nov 2021, accepted 24 Nov 2021

\section{Supplementary material}

To view supplementary material for this article, please visit https://doi.org/10.1192/bjo.2021. 1072.

\section{Data availability}

Following the data policy of the University Hospital of Zurich, the data that support the findings of this study are available on request from the corresponding author (C.H.).

\section{Acknowledgements}

We thank Martina Blaser, coordinator of the cantonal priority programme on suicide prevention, for her inspiring suggestions and comments. We also thank the Cantonal Police Force of Zurich for the correspondence on current suicide statistics.

\section{Author contributions}

C.H., A. Bandli, B.K. and S.O. conceptualised the study and carried out the initial planning. C.H., A. Bandli, A. Bankwitz and A.R. collected the data and constructed the data-set. C.H., M.D.B. and A. Bankwitz carried out the statistical analysis, which was refined by M.D.B, A.R., T.V.D.A. and S.O.. C.H., A. Bankwitz and A. Bandli prepared the first draft of the report, which was revised by S.O., B.K. and E.S. All authors reviewed and contributed to the final draft and approved the final version for publication.

\section{Funding}

There was no specific funding for this study; C.H., A. Bankwitz, A.R., T.V.D.A. and S.O. were supported by the Hans and Marianne Schwyn Foundation and the Foundation for the Promotion of Psychiatry and Psychotherapy, Zurich. The sponsors had no role in the study design, data collection, data analysis, data interpretation or writing of the report.

\section{Declaration of interest}

None declared.

\section{References}

1 Zhu N, Zhang D, Wang W, Li X, Yang B, Song J, et al. A novel coronavirus from patients with pneumonia in China, 2019. N Engl J Med 2020; 382: 727-33.
2 Gilan D, Blessin M, Kunzler A, Stoffers-Winterling J, Müssig M, Yuen KSL, et al. Psychomorbidity, resilience, and exacerbating and protective factors during the SARS-CoV-2-pandemic'a systematic literature review and results from the German COSMO-PANEL. Dtsch Arztebl Int 2020; 117: 625-32.

3 Moccia L, Janiri D, Pepe M, Dattoli L, Molinaro M, De Martin V, et al. Affective temperament, attachment style, and the psychological impact of the COVID19 outbreak: an early report on the Italian general population. Brain Behav Immun 2020; 87: 75-9.

4 Bäuerle A, Teufel $M$, Musche V, Weismüller B, Kohler $H$, Hetkamp $M$, et al. Increased generalized anxiety, depression and distress during the COVID-19 pandemic: a cross-sectional study in Germany. J Public Heal 2020; 42: 672-8.

5 Pappa S, Ntella V, Giannakas T, Giannakoulis VG, Papoutsi E, Katsaounou P. Prevalence of depression, anxiety, and insomnia among healthcare workers during the COVID-19 pandemic: a systematic review and meta-analysis. Brain Behav Immun 2020; 88: 901-7.

6 Mengin A, Allé MC, Rolling J, Ligier F, Schroder C, Lalanne $L$, et al. Psychopathological consequences of confinement. Encephale 2020; 46: S43-52.

7 Sher L. Resilience as a focus of suicide research and prevention. Acta Psychiatr Scand 2019; 140: 169-80.

8 Sher L. The impact of the COVID-19 pandemic on suicide rates. QJM 2020; 113 707-12.

9 Chang S-S, Gunnell D, Sterne JAC, Lu T-H, Cheng ATA. Was the economic crisis 1997-1998 responsible for rising suicide rates in East/Southeast Asia? A timetrend analysis for Japan, Hong Kong, South Korea, Taiwan, Singapore and Thailand. Soc Sci Med 2009; 68: 1322-31.

10 CourtetP, Olie E, Debien C, Vaiva G. Keep socially (but not physically) connected and carry on: preventing suicide in the age of COVID-19. J Clin Psychiatry 2020; 81: 20com13370

11 Milner A, Page A, LaMontagne AD. Long-term unemployment and suicide: a systematic review and meta-analysis. PLOS One 2013; 8: 1-6.

12 Nordt C, Warnke I, Seifritz E, Kawohl W. Modelling suicide and unemployment: a longitudinal analysis covering 63 countries, 2000-11. Lancet Psychiatry 2015; 2: $239-45$

13 Kawohl W, Nordt C. COVID-19, unemployment, and suicide. Lancet Psychiatry 2020; 7: 389-90.

14 International Labor Organization. ILO Monitor. COVID-19 and the World of Work (7th edn). International Labor Organization, 2021 (https://www.ilo.org/wcmsp5/ groups/public/—dgreports/—dcomm/documents/briefingnote/wcms_767028. pdf).

15 State Secretariat for Economic Affairs SECO. Die Lage auf dem Arbeitsmarkt. 2020.

16 Gunnell D, Appleby L, Arensman E, Hawton K, John A, Kapur N, et al. Suicide risk and prevention during the COVID-19 pandemic. Lancet Psychiatry 2020; 7: 468-71.

17 Holmes EA, O'Connor RC, Perry VH, Tracey I, Wessely S, Arseneault L, et al. Multidisciplinary research priorities for the COVID-19 pandemic: a call for action for mental health science. Lancet Psychiatry 2020; 7: 547-60.

18 Reger MA, Stanley IH, Joiner TE. Suicide mortality and coronavirus disease 2019-A perfect storm? JAMA Psychiatry 2020; 77: 1093-4.

19 WHO Suicide Prevention. WHO, no date (https://www.who.int/health-topics/ suicide\#tab=tab_1 [cited 20 April 2021]).

20 Chen S, Jones PB, Underwood BR, Moore A, Bullmore ET, Baneriee S, et al. The early impact of COVID-19 on mental health and community physical health services and their patients' mortality in Cambridgeshire and Peterborough, UK. J Psychiatr Res 2020; 131: 244-54.

21 Pirkis J, John A, Shin S, DelPozo-Banos M, Arya V, Analuisa-Aguilar P, et al. Suicide trends in the early months of the COVID-19 pandemic: an interrupted time-series analysis of preliminary data from 21 countries. Lancet Psychiatry 2021; 8: 579-88.

22 Brülhart M, Lalive R. Daily suffering: helpline calls during the Covid-19 crisis. Covid Econ 2020; 19: 143-58.

23 Penfold RB, Zhang F. Use of interrupted time series analysis in evaluating health care quality improvements. Acad Pediatr 2013; 13 (Suppl. 6): S38-44.

24 Bernal JL, Cummins S, Gasparrini A. Interrupted time series regression for the evaluation of public health interventions: a tutorial. Int J Epidemiol 2017; 46: $348-55$.

25 The AMDP System: Manual for Assessment and Documentation of Psychopathology in Psychiatry. Hogrefe Publishing, 2018.

26 Wolfersdorf M, Etzersdorfer E. Suizid und Suizidprävention [Suicide and Suicide Prevention]. Kohlhammer Verlag, 2013.

27 Bundesamt für Justiz. BBI 20067001 - Botschaft zur Änderung des Schweizerischen Zivilgesetzbuches (Erwachsenenschutz, Personen- und Kindesrecht) [BBI 20067001 - Message on the Amendment of the Swiss Civil Code (Adult Protection, Personal and Child Rights)], 2006. (https://www. fedlex.admin.ch/eli/fga/2006/899/de [cited 12 April 2021)]. 
$28 \mathrm{R}$ Core Team. R: A Language and Environment for Statistical Computing. R Foundation for Statistical Computing, 2021 (http://www.r-project.org/ index.html).

29 Mansfield $K E$, Mathur $R$, Tazare J, Henderson AD, Mulick AR, Carreira $H_{\text {, }}$ et al. Indirect acute effects of the COVID-19 pandemic on physical and mental health in the UK: a population-based study. Lancet Digit Heal 2021; 3: e217-30

30 Cantonal Police Force of Zurich. Annual Report on the Police Crime Statistics, 2020. (https://www.zh.ch/de/sicherheit-justiz/delikte-praevention/kriminalstatistik.html [cited April 29, 2021]).

31 Schweizerisches Gesundheitsobservatiorium. Suizid. OBSAN, 2021 (https:/l www.obsan.admin.ch/de/indikatoren/suizid)

32 Kanton of Zurich: Health Directorate. Suicide Prevention - Projects, no date (https://www.gesundheitsfoerderung-zh.ch/themen/suizidpraevention [cited 19 April 2021]).

33 Deisenhammer EA, Kemmler G. Decreased suicide numbers during the first 6 months of the COVID-19 pandemic. Psychiatry Res 2021; 295: 113623.
34 Hernandez-Calle D, Martinez-Ales G, Mediavilla R, Aguirre P, Rodriguez-Vega B, Bravo-Ortiz MF. Trends in psychiatric emergency department visits due to suicidal ideation and suicide attempts during the COVID-19 pandemic in Madrid, Spain. J Clin Psychiatry 2020; 81: 20113419.

35 Pridmore S, Ahmadi J, Pridmore W. Suicide of Australians during the Vietnam War. Australas Psychiatry 2018; 26: 149-51.

36 Tanaka $\mathrm{T}$, Okamoto $\mathrm{S}$. Increase in suicide following an initial decline during the COVID-19 pandemic in Japan. Nat Hum Behav 2021; 5: 229-38.

37 de Quervain D. The Swiss Corona Stress Study. OSFPreprints [Preprint] 2020. Available from: https://osf.io/jqw6a/ [cited 13 Dec 2021].

38 Schnell $\mathrm{T}$, Krampe $\mathrm{H}$. Meaning in life and self-control buffer stress in times of COVID-19: moderating and mediating effects with regard to mental distress. Front Psychiatry 2020; 11: 983.

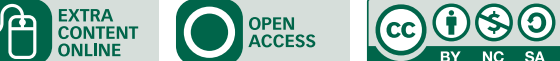

